\title{
Hinweise für den Benutzer
}

Gliederung

I. Zur Stichwortauswahl . . . . . . . . . . . . . . . . . VIII

II. Zum Stichwortansatz . . . . . . . . . . . . . . . . . . . . . . . . IX

III. Zur Definition der Lexeme . . . . . . . . . . . . . . . . . . . . . . XI

IV. Zum Aufbau der Wörterbuchartikel . . . . . . . . . . . . . . . . . . XIV

V. Zu den grammatischen Angaben . . . . . . . . . . . . . . . XVI

VI. Zur Darstellung des Kontextes . . . . . . . . . . . . . . . . . . . . XXI

VII. Zu den stilistischen Kennzeichnungen und Anwendungsbedingungen . . . . XXII

VIII. Zu den Akzent- und Ausspracheangaben . . . . . . . . . . . . . . . . XXIV

IX. Typographisches . . . . . . . . . . . . . . . . . . . XXVII

X. Abkürzungsverzeichnis .. . . . . . . . . . . . . . . . XXIX

\section{Zur Stichwortauswahl}

Das Handwörterbuch erfaßt vorrangig den Teil der Lexik, 'den man zum Zentrum der Allgemeinsprache rechnen darf, der der nichtfachgebundenen Kommunikation dient, weitgehend gruppenunspezifisch ist und weitgehend überregional verwendet wird. Im Gegensatz dazu und außerhalb des Zentrums, an der Peripherie, stehen die Fachwortschätze und diejenigen landschaftlich gebundenen Lexeme (Regionalismen), die in der Allgemeinsprache nicht häufig vorkommen. Die Grenzen zwischen Zentrum und Peripherie sind jedoch fließend. In dem Maße, wie im Alltag Fachgebiete jeweils in das Zentrum des allgemeinen Interesses rücken, wechseln Fachtermini in die Randzone der Allgemeinsprache über. So haben Fachgebiete wie Wirtschaft, Sport, Technik u. a. die Allgemeinsprache wesentlich bereichert.

Der Anteil der einzelnen Fachwortschätze an der Lexik der Allgemeinsprache ist recht unterschiedlich. Dem trägt das Handwörterbuch Rechnung, indem es bei der Stichwortauswahl besonders die Fachgebiete berücksichtigte, die in unserem Alltag eine besondere Rolle spielen.

Bei der Stichwortauswahl wurde der Wortschatz der letzten dreißig Jahre zugrunde gelegt. Damit ist die Aufnahme veralteter Lexeme ausgeschlossen (z. B. Sozietät, Siechenhaus). Aufgenommen wurden lediglich die Lexeme mit der Tendenz zum Veralten (z. B. wohlerzogen). Historische Lexeme, d. h. Lexeme, die Erscheinungen vergangener Epochen widerspiegeln, wurden unter dem Gesichtspunkt ihrer Gewichtigkeit ausgewählt (z. B. Sklave). Die Auswahl ideologierelevanter Stichwörter folgt den allgemeinen Prinzipien der Stichwortauswahl, doch finden in diesem Bereich bestimmte Lexeme Berücksichtigung, die nach 1945 erst neu entstanden und heute bereits veraltet sind. Es handelt sich dabei um Lexeme, die bestimmte Etappen der jüngeren historischen Entwicklung widerspiegeln und für die Darstellung dieser Zeit weiterhin Verwendung finden. Es sind dies Wörter wie Neubauer, Neulehrer, Arbeiter- und -Bauern- 